\title{
Life history and matrix heterogeneity interact to shape metapopulation connectivity in spatially structured environments
}

\author{
Jeffrey S. Shima, ${ }^{1,3}$ Erik G. Noonburg, ${ }^{2}$ and Nicole E. Phillips ${ }^{1}$ \\ ${ }^{1}$ School of Biological Sciences and the Victoria University Coastal Ecology Lab (VUCEL), P.O. Box 600, \\ Victoria University of Wellington, Wellington, New Zealand 6140 \\ ${ }^{2}$ Department of Biological Sciences, Florida Atlantic University, 2912 College Avenue, Davie, Florida 33314 USA
}

\begin{abstract}
Metapopulation models have historically treated a landscape as a collection of habitat patches separated by a matrix of uniformly unsuitable habitat. This perspective is still apparent in many studies of marine metapopulations, in which recruitment variation is generally assumed to be primarily the result of variability in ocean currents and interactions with disperser behavior, with little consideration of spatial structure that can affect disperser viability. We use a simple model of dispersal of marine larvae to demonstrate how heterogeneity in dispersal habitat (i.e., the matrix) can generate substantial spatial variation in recruitment. Furthermore, we show how this heterogeneity can interact with larval life-history variation to create alternative patterns of source-sink dynamics. Finally, we place our results in the context of spatially structured matrix population models, and we propose the damping ratio of the connectivity matrix as a general and novel measure of landscape connectivity that may provide conceptual unification to the fields of metapopulation biology and landscape ecology.

Key words: connectivity matrix; damping ratio; dispersal; landscape connectivity; larval life history; matrix heterogeneity; metapopulation connectivity.
\end{abstract}

\section{INTRODUCTION}

Fragmented populations may be interconnected by dispersal of organisms, and resulting "connectivity" shapes metapopulation dynamics (Hanski 2002), rates of evolution (Hanski and Gaggiotti 2004), and efficacy of management and conservation strategies (Saunders et al. 1991). Dispersal among fragmented populations has often been modeled as linear movements through a binary landscape (e.g., MacArthur and Wilson 1967, Doak et al. 1992, Hanski 2002). Within this simplified conceptual framework, fragmented populations exist within patches of suitable habitat separated from one another by a matrix of non-habitat. Nonetheless, most ecological landscapes are not binary, and many organisms disperse through a heterogeneous matrix that can affect the fitness of dispersers (Roff 1977, Elkin and Reid 2005, Matter 2006, Shima and Swearer 2009), probability of successful dispersal (Ricketts 2001, Vandermeer and Caravajal 2001, Revilla et al. 2004), and thus, patterns of connectivity.

Although the effect of landscape heterogeneity on dispersal and demographic connectivity is an active area of research (e.g., Cronin 2007, Vos et al. 2007, Goodsell and Connell 2008, Vandermeer and Lin 2008) some important implications remain elusive, in part because

Manuscript received 5 November 2008; revised 5 August 2009; accepted 7 August 2009. Corresponding Editor: M. Fortin.

${ }^{3}$ E-mail: Jeffrey.Shima@vuw.ac.nz
(1) we sometimes fail to appreciate (and hence study) the potential effects of landscape heterogeneity and (2) we may lack general analytical frameworks that enable us to link landscape heterogeneity with measures of metapopulation connectivity. The aim of our paper is thus twofold: first, we use a simple model of dispersal to highlight potentially important and novel implications of landscape heterogeneity for marine-based metapopulations. Second, we build upon our simple model to develop and illustrate a new approach that facilitates a system-wide estimate of metapopulation connectivity in heterogeneous landscapes.

In coastal marine ecosystems, many species exist as patchy populations that are naturally fragmented by landscape features. Benthic organisms that inhabit discontinuous rocky reef habitats are typically isolated by expansive sandy beaches and embayments. Moreover, adult stages of many marine organisms are relatively sedentary and produce large numbers of offspring that develop as larvae in offshore coastal waters for extended periods lasting weeks to months (Roughgarden et al. 1985, Caley et al. 1996, Kritzer and Sale 2004). Larval dispersal is the primary mechanism for connecting and replenishing fragmented populations in marine settings, and variation in larval supply (a function of larval survival and delivery) into local populations is thought to be an important determinant of population dynamics and structure (Caley et al. 1996, Kritzer and Sale 2004, Pineda et al. 2007). 
The coastal ocean matrix through which marine larvae disperse is chemically, physically, and biologically variable (Cushing 1975, Lasker 1981, Denman and Powell 1984, Steele 1989, Mann and Lazier 1996). Furthermore, dispersal of marine larvae is heavily influenced by physical forcing: the ocean matrix is constantly moving, and a substantial fraction of larval production may be lost due to hydrodynamic advection away from suitable habitats (Hjort 1914, Largier 2003). Other sources of heterogeneity in the coastal ocean matrix (e.g., patchiness of food, predators, or stressors) may influence fitness of dispersers by altering phenotypic traits and/or survival probabilities (Hjort 1914, Meekan et al. 2003, Shima and Swearer 2009).

Most recent models of marine larval dispersal and metapopulation connectivity focus on hydrodynamically mediated dispersal (e.g., diffusion, advection, and retention of larvae by currents and eddy structures; Gaines et al. 2003, Largier 2003, Cowen et al. 2006, Aiken et al. 2007, Mitarai et al. 2008, Siegel et al. 2008) and larval behaviors that potentially modulate advective and diffusive processes (Paris et al. 2007), ignoring other sources of matrix heterogeneity. Although these models provide powerful and exciting insights into potential patterns of larval dispersal and connectivity in marine metapopulations, most make predictions based upon assumptions of a spatially unstructured matrix (albeit, a moving one). The effect of matrix heterogeneity (other than water movement) on connectivity within marine metapopulations remains largely unexplored.

Here, we use a simple model of random dispersal among coastal subpopulations to demonstrate the potential for spatially structured sources of larval mortality (a form of matrix heterogeneity) to generate spatial structure in larval settlement in the absence of any complex hydrodynamic forcing. Our modeling framework is informed and motivated by our empirical work (Phillips and Shima 2006) on sea urchins and abalone, which are of great ecological and economic importance (Morgan and Shepherd 2006). Urchins and abalone that inhabit rocky reefs exemplify the life history patterns of many marine reef organisms. They are patchily distributed across their range, as a set of discrete local populations that are potentially connected by dispersing larvae (reviewed in Morgan and Shepherd 2006). Our previous work (Phillips and Shima 2006) suggests that the larvae of these two species are differentially affected by suspended sediments from river outfalls (i.e., a form of spatially structured matrix heterogeneity). Specifically, we found that the dispersing larvae of urchins have constant low vulnerability to sediments through larval ontogeny, whereas abalone larvae experience initially high vulnerability that diminishes with larval age (possibly due to differences in traits such as larval morphology, maternal provisioning, developmental patterns; Phillips and Shima 2006). Hence, we evaluate effects of matrix heterogeneity across a range of larval "vulnerabilities" bracketed by these empirical observations.

We simulate larval dispersal to explore how vulnerability interacts with other larval traits and changing environmental conditions (e.g., Airoldi 2003, Thrush et al. 2004) to influence population processes at two scales. First, we examine predictions for dispersal, settlement, and source-sink dynamics at the scale of local subpopulations. Second, we use the results of our dispersal model to analyze landscape-level properties at the scale of the entire metapopulation. More specifically, the outcome of simulating dispersal among subpopulations is a connectivity matrix, which describes the probabilities of moving among subpopulations (e.g., Largier 2003, Cowen et al. 2006). Connectivity matrices naturally fall within the general framework of spatially structured matrix population models, for which an extensive body of theory already exists (see Caswell 2001:62-71). This theory provides analytic techniques to derive measures of the population-level consequences of individual dispersal behavior. We use one of these measures, the damping ratio (sensu Caswell 2001:95100), in a new way: the damping ratio of a connectivity matrix serves as a summary measure of metapopulation (or landscape) connectivity. This measure may help unify historically divergent perspectives on connectivity from the fields of landscape ecology and metapopulation biology (e.g., Tischendorf and Fahrig 2000, 2001, Moilanen and Hanski 2001, Bode et al. 2008).

\section{Models \\ Modeling larval dispersal and spatially structured mortality}

Dispersal of pelagic larvae depends on advection and turbulence at multiple scales, as well as active larval behavior (e.g., vertical migrations through the water column or responses to settlement cues from nearby reefs). This dispersal process has frequently been modeled in an advection-diffusion framework by simulating movement of larvae as particles in a flow field with a constant mortality rate (Possingham and Roughgarden 1990, Gaylord and Gaines 2000, Largier 2003, Kaplan 2006). We simulate larval dispersal along a coastline as a one-dimensional random walk, which is a discrete approximation to advection/diffusion in continuous time and space (Okubo and Levin 2001). This is clearly a simplistic representation of dispersal (effectively a mean of many stochastic settlement events; e.g., Siegel et al. 2008); however, this simple framework allows us to study the effects of spatially structured sources of stress and mortality, and their interaction with larval life history features, in the absence of hydrodynamic forcing.

The simulated coastline is divided into $L$ length units, with $M$ equally spaced units that contain mortality sources (e.g., coastal discharges of suspended sediments). The duration of the larval stage is $T$ time steps. At each time step after fertilization, a larva can move 
one unit to the left, one unit to the right, or stay put with probabilities $p_{\mathrm{l}}, p_{\mathrm{r}}$, and $1-\left(p_{1}+p_{\mathrm{r}}\right)$, respectively. We assume periodic boundaries such that a larva at location $L$ that moves one step to the right arrives at location 1 . The movement path of a larva is therefore a sequence of $T$ adjacent or identical locations (i.e., a larva cannot skip over any points in its trajectory). At any step, the probability of death upon encountering a mortality source is $m(t)$, which depends on larval age, $t=1,2, \ldots$, $T$. Since probability of death is variable, hereafter we refer to the $M$ sources of mortality as spatially structured "stressors."

The random walk of $T$ time steps results in a dispersal kernel, which is the probability that a larva born at location $i$ arrives at location $j$ (Neubert et al. 1995). Similarly, the subpopulation at each location $i$ has an accumulation kernel (sensu Aiken et al. 2007; i.e., the probability that a larva that settles at $i$ originated at location $j$ ). In the absence of spatially structured stressors, the dispersal and accumulation kernels are independent of location; moreover, if $p_{1}=p_{\mathrm{r}}$, the dispersal and accumulation kernels are symmetric and identical to one another. We can simulate simple advection by $p_{1} \neq p_{\mathrm{r}}$, in which case the dispersal and accumulation kernels are skewed but differ from one another only in the direction of skew (i.e., they are mirror images of one another). The addition of spatially structured stressors modifies the distribution of surviving settlers ("realized" dispersal and accumulation kernels). The realized dispersal and accumulation kernels may differ from one another and they may differ among locations, as we show in the next section.

We employ a simple model of larval vulnerability to stressors to caricature the range of life history variation observed in our empirical work (Phillips and Shima 2006). In the simplest case of a species in which vulnerability does not change with larval age, $m(t)=\mu$ is constant for all $t$, where $\mu$ is stressor strength. We use a step function to simulate decreasing vulnerability with age, and we impose the condition that the sum of $m(t)$ over all $t$ is equal to $\mu T$. For species in which larvae are vulnerable for the initial fraction $v$ of the dispersal stage, we set $m(t)=\mu / v$ for $t<v T$ and $m(t)=0$ for the remaining $t \geq v T$, and we vary $v$ in the range $\mu \leq v \leq 1$. The form of vulnerability implies that an individual that encounters a mortality source at random during the larval stage has, on average, the same probability of death regardless of life history type, characterized by $v$. In our simulations, we vary $v$ in increments of size $T^{-1}$ to avoid mortality during fractions of time steps due to the discretization.

We simulate $N$ larvae originating at each location to generate a connectivity matrix for the population. The connectivity matrix, $\mathbf{C}$, is composed of elements $c_{i j}$, the proportion of larvae arriving at location $i$ that originated at location $j$. The columns of the connectivity matrix are therefore the realized dispersal kernels for the larvae originating at each location $j$, and the rows are the realized accumulation kernels for the subpopulation at each location $i$.

\section{Matrix model representation and analysis}

The dispersal and mortality processes in our simulations can be rewritten in a stochastic matrix model framework using the same movement and survival rules and assumptions described in the previous section. The movement probabilities for each time step $t$ in the dispersal stage comprise a matrix $\mathbf{D}$, with element $d_{i j}$ as the probability of moving from location $j$ to location $i$ during any time step. In our model, all elements on the diagonal are equal to the probability of staying put: $d_{i i}=$ $1-\left(p_{1}+p_{\mathrm{r}}\right)$. Each element above or below the diagonal $\left(d_{i j}\right.$ for $i=j-1$ and $\left.i=j+1\right)$ is set to $p_{1}$ or $p_{\mathrm{r}}$, respectively. With our assumption of periodic boundaries, $d_{1 L}=p_{\mathrm{r}}$ and $d_{L 1}=p_{1}$. Similarly, we define the survival matrix, $\mathbf{S}(t)$, to be a diagonal matrix with element $s_{i i}(t)$ as the probability of survival if a larva moves to location $i$ during time step $t$, regardless of where it moved from. In our simulations, we assume the spatially structured stressors are the only sources of mortality. Hence, $s_{i i}(t)=$ $1-m(t)$ for $i$ in each of the $M$ stressor sources, and $s_{i i}(t)$ $=1$ otherwise. For a species with constant vulnerability throughout the dispersal stage, $\mathbf{S}(t)$ is independent of time, with diagonal elements $s_{i i}(t)=1-\mu$. For species with a vulnerable period, $s_{i i}(t)=1-\mu / v$ for $t<v T$ and $s_{i i}(t)=1$ for $t \geq v T$. The connectivity matrix is the result of iterating the one-step survival and dispersal matrix over all $T$ steps in the dispersal stage:

$$
\mathbf{C}=\prod_{t=1}^{T} \mathbf{S}(t) \mathbf{D} .
$$

The connectivity matrix projects the spatial distribution of settlers from a population with any given initial spatial distribution. For example, suppose (1) the initial population of reproducing adults is $n_{0}$, a vector of length $L$ in which the $i$ th element is the number of adults at location $i$ on the coastline; (2) reproduction occurs at discrete intervals, and settlers mature by the next reproductive event; and (3) survival and reproduction are constant and density independent. With these assumptions, the distribution of new recruits to the reproducing population is $n_{1}=b \mathbf{C} n_{0}$, where $b$ is the product of per capita larval production and survival from settlement to reproduction. The distribution of offspring of these recruits is $n_{2}=b \mathbf{C} n_{1}=b^{2} \mathbf{C}^{2} n_{0}$, and the distribution of offspring of the initial $n_{0}$ adults in the $k$ th interval is $n_{k}=b^{k} \mathbf{C}^{k} n_{0}$. Here, we have ignored survival and fecundity of (sedentary) adults over successive reproductive events (i.e., we treated the species as semelparous). This allows us to analyze the consequences of dispersal, embodied in the properties of $\mathbf{C}$, in isolation from dynamics within subpopulations (e.g., density-dependent survival, growth, and the like).

We apply two general results from matrix theory to describe the population-level consequences of individual 
dispersal behavior and mortality. First, under the assumptions listed in the previous paragraph, $n_{k}$ approaches a stable spatial distribution (i.e., the subpopulation sizes may differ from one another, but their relative sizes approach values that are constant over time) that is independent of the initial spatial distribution $n_{0}$. The stable spatial distribution is equal to the right eigenvector $\left(w_{1}\right)$ of $\mathbf{C}$ (Caswell 2001:79-86). Second, the rate at which the population approaches the stable spatial distribution is equal to the ratio of the largest to the second largest eigenvalue of $\mathbf{C}$ :

$$
\rho=\frac{\lambda_{1}}{\left|\lambda_{2}\right|}
$$

where $\left|\lambda_{2}\right|$ denotes the modulus of $\lambda_{2}$, i.e., for a complex eigenvalue $\lambda_{2}=a+b i,\left|\lambda_{2}\right|=\sqrt{\left(a^{2}+b^{2}\right)}$. The ratio $\rho$ is termed the damping ratio (Caswell 2001:95-96), and it varies from a minimum of 1 to infinity.

The damping ratio provides an intuitive quantitative measure of population-level connectivity: large $\rho$ implies that dispersers rapidly spread throughout existing patches of habitat, whereas small $\rho$ implies increasingly isolated patches. For example, a population with large $\rho$ that is perturbed from its stable spatial distribution (e.g., by extinction of some subpopulations) will rapidly return to the stable distribution via dispersal among the subpopulations. Alternatively, if a group of empty habitat patches were inoculated with a few individuals, $\rho$ is the rate at which successive generations of offspring of the initial propagule would fill the patches on the approach to the stable spatial distribution.

\section{RESULTS}

\section{Effects of matrix heterogeneity and larval life history on dispersal and settlement}

Matrix heterogeneity (i.e., spatially structured sources of larval stress) affects the probability of larval survival during the dispersal period, and thus alters realized patterns of dispersal and connectivity among local populations (Fig. 1 shows the comparison between column I with columns II and III). Moreover, larval vulnerability mediates the effects of spatially structured stressors. Larvae that are vulnerable to stressors throughout their larval ontogeny (Fig. 1, column II) have a reduced probability of surviving a dispersal trajectory that takes them past point sources of stress regardless of larval age. The result is a "fragmented" connectivity matrix (Fig. 1B) comprised of some local populations (those furthest from point sources of stress) that produce and accumulate most of the successful dispersers (Fig. 1E), and other local populations (at or near sources of stress) that produce and accumulate relatively few (Fig. 1H).

Realized accumulation kernels summarize the distributions of origins of successful dispersers (i.e., larvae that survive and settle) to a given location. Likewise, realized dispersal kernels summarize the distributions of destinations of all successful dispersers produced at a given location. Local populations that produce larvae with a constant vulnerability to stressors (or those embedded within a spatially homogeneous matrix) exhibit accumulation kernels that are identical to the dispersal kernels regardless of their location (Fig. 1, columns I-II). Local populations embedded within a spatially heterogeneous matrix and connected by dispersal of larvae that are vulnerable to stressors only early in ontogeny (Fig. 1, column III) have accumulation kernels that differ from the dispersal kernels. This asymmetry arises because larvae that originate at or near a source of stress tend to encounter stressors at an earlier developmental stage (i.e., when they are most vulnerable), whereas larvae that originate farther from stressors are more likely to outgrow their vulnerability, and thus pass by a stressor relatively unscathed. Consequently, larvae that originate far from sources of stress exhibit a realized dispersal kernel that is approximately Gaussian in form (as per spatially homogeneous stressors, cf. Fig. $1 \mathrm{D}, \mathrm{F}$, lower panels) and an accumulation kernel that is "punctuated" at point sources of stress (Fig. 1F, upper panel). Populations at or near sources of stress may receive relatively high numbers of successful dispersers (originating from a range of distant populations), though they contribute relatively few successful dispersers to their own or more distant populations (Fig. 1I).

\section{Consequences of dissimilar dispersal and accumulation kernels: sinks and sources}

For any given location, the ratio of total disperser accumulation (summed over the entire realized accumulation kernel) to total production of successful dispersers (summed over the entire realized dispersal kernel) gives a measure of "net cost" of a local population to the wider metapopulation. We present the net cost as the logarithm of this ratio such that a net cost of 0 implies production equal to accumulation, and a net cost $>0$ implies accumulation is greater than production.

Net costs are 0 when dispersers diffuse though a uniform matrix (Fig. 2A, black line) or when dispersers with constant vulnerability to stress diffuse through a heterogeneous matrix (Fig. 2A, gray line). When dispersers with early larval vulnerability disperse through a heterogeneous matrix, however, some local populations contribute disproportionately to the pool of successful dispersers available to the wider metapopulation and therefore become important sources (sensu Nee 2007) for the metapopulation. In contrast, the interaction of stage-structured vulnerability and spatially structured stressors causes other populations to become sinks (Fig. 2A, dotted line). The difference in predicted patterns of net costs for the two life histories occurs despite equivalent stable spatial distributions (Fig. 2B, dotted and gray lines).

We simulated dispersal of an equal number of larvae originating from each local population in order to isolate the effects of dispersal from the population 
I) Homogenous stressors
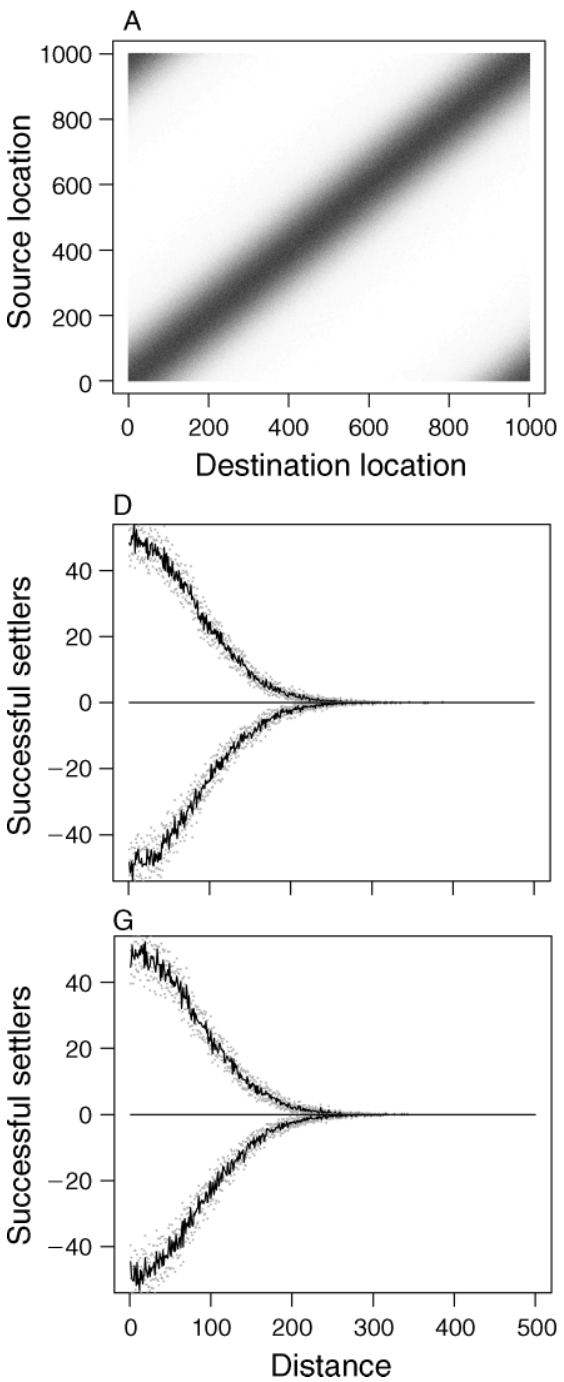

II) Spatially structured stressors and constant vulnerability

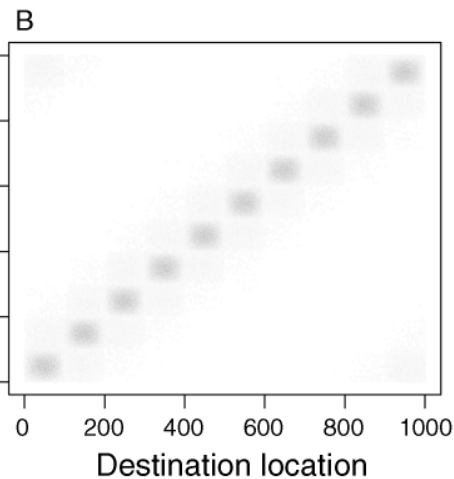

E
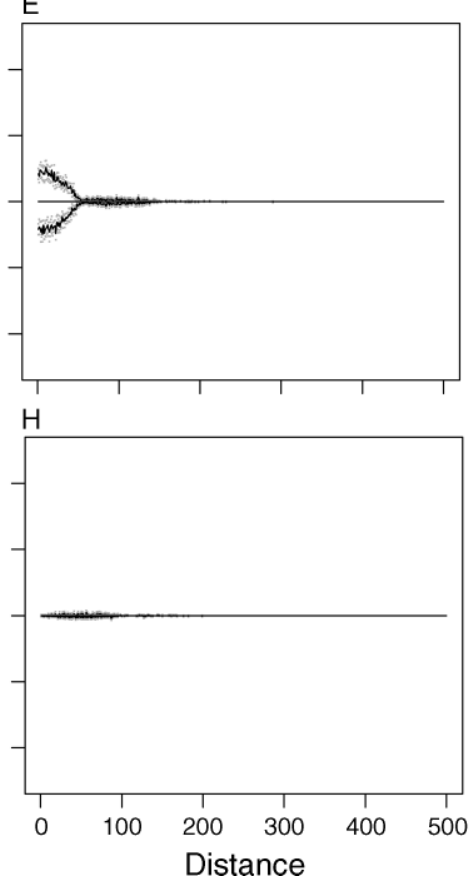

III) Spatially structured stressors and early vulnerability

C

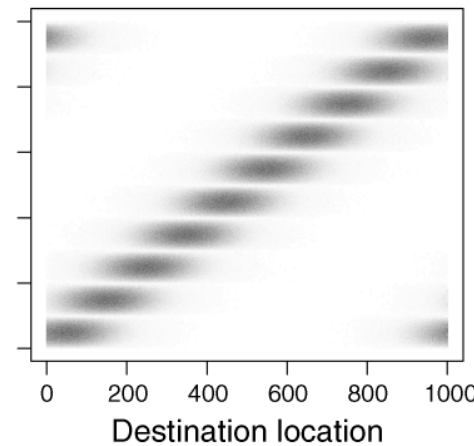

$\mathrm{F}$

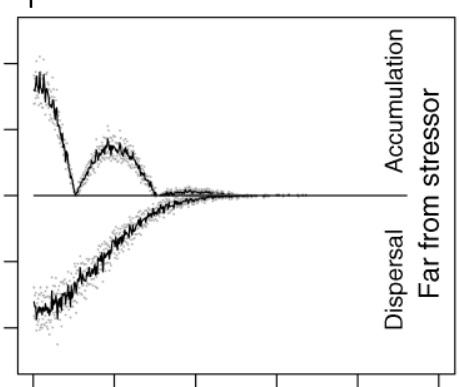

।

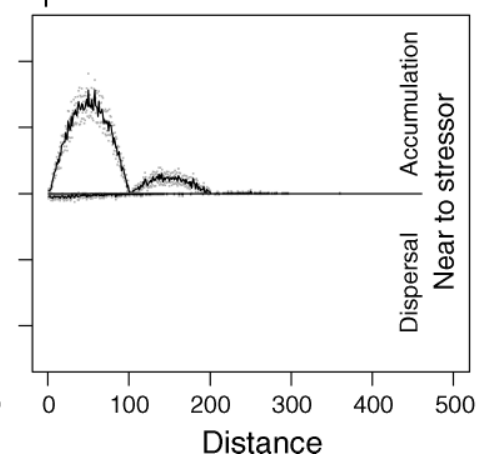

FIG. 1. Patterns of connectivity for random walk simulations of larval dispersal along a coastline of length $L=1000$ units with 10 equally spaced stressors (at locations $1,101,201, \ldots, 901$ ). A population in each length unit releases $N=10000$ larvae that move to the left or right with equal probability $\left(p_{1}=p_{\mathrm{r}}=0.333\right)$ for $T=10000$ time steps. Panels in each column contain output from three mortality scenarios: column I, no spatially structured stressors (probability of death $[m(t)]=0$ for all larval ages $[t]$ ); column II, larvae with constant vulnerability to spatially structured stressors $[m(t)=0.2$ for all $t$ ]; column III, larvae with early vulnerability to spatially structured stressors $[m(t)=1$ for $t<v T, m(t)$ for $t \geq v T, v=0.2$, where $v$ is the vulnerability of the initial fraction of the dispersal stage]. Row 1 shows connectivity matrices, $\mathbf{C}$, with darker shading indicating larger $c_{i j}$, the proportion of larvae arriving at location $i$ that originated at location $j$ (lighter shading in panel $\mathrm{B}$ indicates lower overall survival). Row 2 shows realized accumulation and dispersal kernels for populations at locations farthest from stressors. Row 3 shows realized accumulation and dispersal kernels for populations at locations that contain stressors. Realized accumulation kernels summarize the distributions of origins of successful dispersers (i.e., larvae that survive and settle) to a given location. Realized dispersal kernels summarize the distributions of destinations of all successful dispersers produced at a given location. The upper half of each panel shows the natal origins for the dispersers that settled either far from a stressor (for the second row) or near a stressor (for the third row), i.e., top halves show where larvae settling to a given site came from. The bottom half (shown as negative numbers) gives destinations of dispersers that started either far from or near to a stressor, i.e., where larvae produced at a given site went. Dispersal kernels are reflected over the horizontal axis for ease of presentation; negative numbers indicate dispersers that moved away from a subpopulation. Mean (solid traces) and 95\% CIs (gray dots) are given for all kernels.

dynamics. However, the number of larvae produced by a local population will, in general, depend on the local population size. Hence, the source-sink dynamics described in the previous paragraph represent only the potential costliness of local populations (e.g., for the case in which local populations have identical numbers of adults). The actual cost of a local population will depend on demographic processes that may vary 

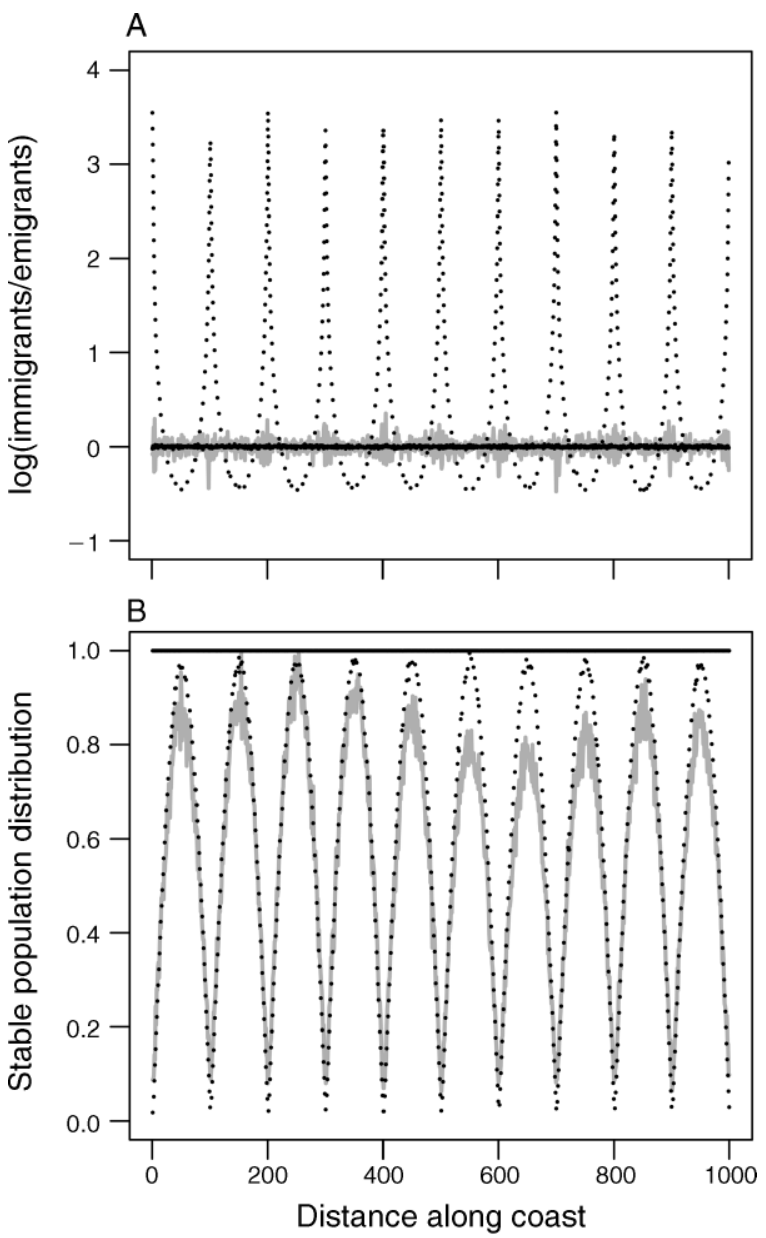

FIG. 2. (A) Net cost of each local population to the metapopulation estimated as the ratio of larval accumulation to larval production (i.e., sink strength) and (B) stable population distribution expressed as a fraction of the largest subpopulation in each simulation. Given are patterns for larval dispersers with early vulnerability to stressors (black dotted lines), constant vulnerability to stressors (gray solid lines), and no spatial stressors (black solid lines), using output from simulations presented in Fig. 1.

spatially, such as density dependence and adult lifespan, in addition to the local settlement rate. Similarly, the stable spatial distributions would be modified by density dependence in the local populations.

\section{Linking subpopulation properties to system-wide connectivity}

The connectivity matrix $(\mathbf{C})$ represents the strength of all point-to-point connections in a metapopulation, and simple patterns of spatial structure may be discerned directly from C. For example, in the simple linear habitat that we simulated, Fig. 1B clearly shows a metapopulation split into several partially isolated clusters of subpopulations. However, in metapopulations with more complex spatial structure, extracting such patterns by eye may be difficult and subjective.
The damping ratio obtained from $\mathbf{C}$ provides a quantitative measure of overall connectivity that can be used for comparisons across systems and to examine the impact of environmental changes. We present four examples to demonstrate how life history features interact with spatially structured mortality to influence connectivity as measured by the damping ratio (Fig. 3). In each example, we calculate the damping ratio over a range of vulnerabilities, $v$ (bracketed by the values used in Fig. 1B, C), as another factor is varied. The four factors that we examine are larval stage duration $(T)$, advection (difference between $p_{1}$ and $p_{\mathrm{r}}$ ), stressor strength $(\mu)$, and stressor extent. We vary stressor extent by increasing the width of each of the $M$ mortality sources to more than one length unit. We show how the changes in the damping ratio are consistent with qualitative predictions for changing system-wide connectivity.

We expect increasing total larval stage duration $(T)$ will increase system-wide connectivity, i.e., connectivity increases with increasing mean distance traveled by larvae. Indeed, this effect is apparent in the damping ratio ( $\rho$; Fig. 3A). The increase in $\rho$ is more rapid for species in which a smaller fraction $(v)$ of the early larval stage is vulnerable because most additional encounters with stressors occur after the end of the vulnerable period.

The damping ratio reveals a strong interaction between advection and vulnerability in their combined effect on system-wide connectivity (Fig. 3B). Increasing advection (the difference between $p_{1}$ and $p_{\mathrm{r}}$ ) increases connectivity when larvae are vulnerable throughout the dispersal stage but decreases connectivity when larvae are vulnerable to mortality only during the first few time steps (small v). With strong advection, larvae are unlikely to stay near their location of natal origin for long. Advection therefore forces more larvae originating upstream of a mortality source through the mortality source early in the larval stage. For larvae with early vulnerability, the effect of increasing mortality is greater than the effect of increasing mean dispersal distance. For larvae with constant vulnerability, however, the effect of advection on survival of individuals originating near a mortality source is small compared to the increase in mean dispersal distance with increasing advection.

Not surprisingly, increasing stressor strength reduces connectivity: the damping ratio decreases as clusters of populations between stressors are increasingly isolated by reducing the probability of survival of dispersers that encounter stressors, and this effect does not differ much across the range of vulnerability (Fig. 3C). Increasing stressor extent causes a greater reduction in connectivity in species with constant vulnerability relative to species with early vulnerability (Fig. 3D). Greater stressor extent increases the expected amount of time a larva spends in the vicinity of stressors; however, this is irrelevant for larvae that have passed the vulnerable portion of the dispersal stage. 

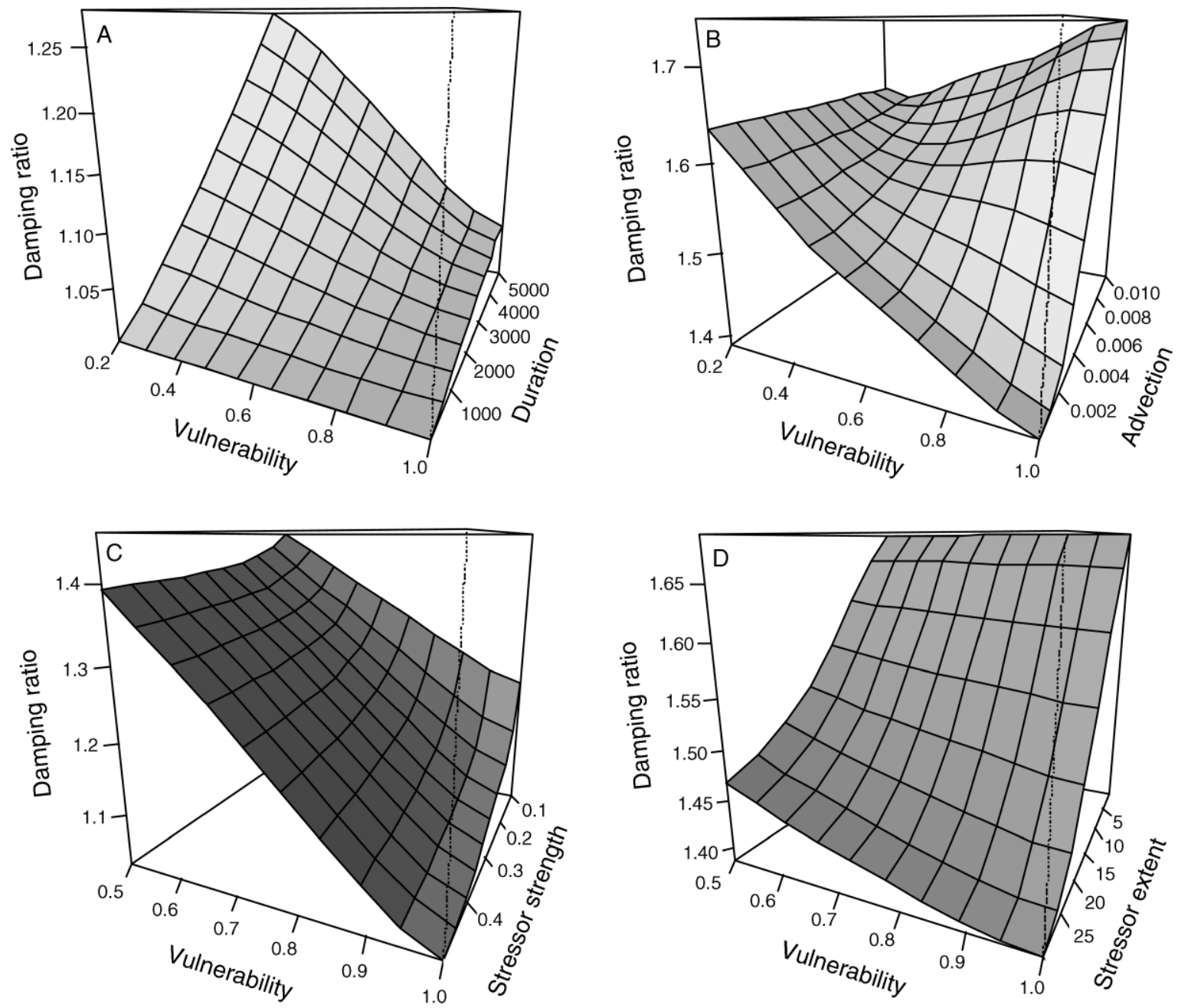

FIG. 3. Metapopulation connectivity response surfaces. Connectivity is estimated by the damping ratio and is presented as a function of larval vulnerability, $v$, to spatially structured stressors and, on the duration axis: (A) larval duration, $T$; (B) strength of advection, $p_{1}-p_{\mathrm{r}}$; (C) strength of stressor, $\mu$; and (D) spatial extent of stressor. Simulated coastline length is $L=500$ units with five equally spaced stressors ( $M$, sources of mortality). Surfaces are shaded for ease of visual interpretation; grayscale does not represent quantitative information.

\section{Discussion}

The intervening landscape (i.e., the matrix) of a metapopulation can affect probabilities of dispersal and successful establishment into a local patch. The role of the matrix is increasingly a focus of study, particularly as landscapes become modified by anthropogenic activities. Emerging evidence from a range of systems tells us clearly that the matrix matters for metapopulation connectivity (Ricketts 2001, Vandermeer and Caravajal 2001, Haynes and Cronin 2004, Murphy and Lovett-Doust 2004, Revilla et al. 2004, Cronin 2007, Vos et al. 2007, Goodsell and Connell 2008, Vandermeer and Lin 2008).

Using a simple representation of larval movement along a coastline, we demonstrated that matrix heterogeneity can generate spatial variation in settlement, and that matrix heterogeneity can interact with larval life history such that the effect on settlement is likely to differ between species (Fig. 1). Our results suggest that local recruitment depends not only on hydrodynamic forcing (the focus of most marine metapopulation simulations), but also the quality of the environment through which larvae move. Furthermore, the interaction between life history and matrix heterogeneity leads to differences in the source-sink status of subpopulations that are not apparent from the spatial pattern of population abundance (Fig. 2). Hence, we suggest that the impacts of matrix quality on dispersal success must be considered along with local habitat quality and demographic rates in subpopulations to understand source-sink structure in a metapopulation.

Our modeling framework was motivated by our past empirical work on larval abalone and sea urchins, and their respective vulnerabilities to suspended sediments 
that enter coastal environments (i.e., the dispersal matrix) from point sources such as rivers and storm drains (Phillips and Shima 2006). In our model, dispersers with early vulnerability to sediments are similar to abalone larvae, while those with constant vulnerability represent sea urchin larvae. We predict that the effects of spatially structured stressors differ between the two species (i.e., effects differ among life histories). Consequently we would expect the introduction of spatially structured stressors to strongly alter spatial covariance in the distributions of these two species. As both urchins and abalone are important grazers of structurally important macroalgae on temperate rocky reefs (Morgan and Shepherd 2006), altered connectivity regimes that result in different population distributions may have consequences for the structure and functioning of local ecosystems. Similarly, as both abalone and urchins are harvested commercially in many areas, altered connectivity regimes and the emergent sourcesink properties of the two species would likely have implications for management, including optimal placements of marine reserves.

We used the matrix formulation of our dispersal model to explore other sources of variation in connectivity, and we asked how these interact with larval vulnerability in a spatially heterogeneous matrix (Fig. $3)$. We chose to explore variation in pelagic larval duration because this is an important life-history trait that varies across many species and it is generally assumed to affect patterns of connectivity (e.g., Caley et al. 1996, Kinlan and Gaines 2003, Teske et al. 2007). Similarly, we chose to explore variation in the rate of advection as a simple proxy for hydrodynamic forcing and/or larval behavior (e.g., directed swimming) - both elements have been the primary focus of recent modeling efforts to explore and quantify connectivity in marine settings (e.g., Cowen et al. 2006, Aiken et al. 2007, Paris et al. 2007, Mitarai et al. 2008, Siegel et al. 2008). We simulated different forms of environmental change by separately varying stressor strength (e.g., sediment loads transported into coastal environments) and stressor extent (e.g., the number or concentration of sediment discharges into a coastal environment). These model explorations provide a useful set of predictions of how connectivity regimes might vary across differing disperser life histories and/or under changing environmental conditions. More generally, our analysis demonstrated the potential for a wide range of variation among species in response to any particular environment. Hence, we suggest that by ignoring larval life-history variation and its interaction with matrix heterogeneity, models that simply treat larvae as particles dispersed by water movement (and/or larval behavior) neglect potentially important sources of variation in metapopulation connectivity.

An additional goal of our model exploration was to demonstrate the utility of standard matrix modeling techniques, and the damping ratio specifically, in the study of connectivity. Given that models of metapopulation and landscape dynamics generally begin with or result in a description of pair-wise connections between habitat patches (i.e., a connectivity matrix), techniques borrowed from matrix modeling approaches provide a natural framework for analysis. Ecologists frequently consider matrix modeling techniques in the context of populations structured by life-history stages (age, size, reproductive status, and the like); however, the framework is generally applicable to populations structured by other factors, including space. Indeed, matrix modeling techniques, including the damping ratio, have been used by others predict and explore spatial structure (e.g., Lebreton and Gonzalez-Davila 1993, Lebreton 1996, Westerberg and Wennergren 2003, Westerberg et al. 2005).

We emphasized the utility of the damping ratio of the connectivity matrix because it links local to landscape processes in a single summary measure, and it complements other measures of connectivity that are used in landscape ecology (reviewed in Calabrese and Fagan 2004). In particular, measures derived from graph theory have been promoted recently for general use in landscape and conservation applications (Urban and Keitt 2001, Minor and Urban 2008, Treml et al. 2008, Urban et al. 2009). Graph theoretic measures of connectivity generally fall in one of two categories: measures based on presence/absence of links between patches and measures that sum patch characteristics or pair-wise connection strengths over all patches. For example, Urban and Keitt (2001) define five measures of landscape connectivity, three of which fit in the first category: number of "components" (groups of connected patches), "order" (number of patches in the largest component), and "diameter" of the largest component (maximum of the minimum number of connections that must be traversed between any pair of patches). These three measures depend on the presence or absence of connections, but ignore connection strengths. The other two measures defined by Urban and Keitt (2001) fit in the second category: "recruitment" (sum of patch sizes weighted by patch quality for the focal species), and "dispersal flux" (sum of patch sizes weighted by patch quality and by probability of dispersal away from each patch). By summing values over all patches, connectivity measures in this category ignore the overall spatial arrangement of patches (e.g., dispersal flux could be high because a few patches are strongly connected with one another but concentrated in one small part of the landscape, or because most patches in the landscape have moderately strong connections).

More recent discussions of landscape connectivity (e.g., Urban and Keitt 2001, O’Brien et al. 2006, Saura and Pascual-Horta 2007, Bode et al. 2008, Minor and Urban 2008) have broadened the list of potential connectivity measures (e.g., to treat clustering, distribution of number of connections, or mean path strength). These measures similarly focus on summarizing num- 
bers of connections or averaging connection strengths over patches. Their utility and appeal stems in part from the intuitive link between the mean flux or number of steps required to move between patches, and individual movement behavior for a given species. Although perhaps less intuitive, the damping ratio avoids problems inherent in many alternative connectivity measures. In particular, the damping ratio integrates information on all pair-wise connection strengths as well as the spatial arrangement of patches.

The definition of landscape connectivity has engendered considerable debate between landscape ecologists and metapopulation ecologists, who have historically viewed connectivity as a purely patch-level property (Hanski 2002). These divergent perspectives on connectivity have hindered exchange of ideas and limited integration of metapopulation biology and landscape ecology (e.g., Tischendorf and Fahrig 2000, 2001, Moilanen and Hanski 2001). A fundamental criticism of the landscape approach is the lack of a clear theoretical link between landscape connectivity and the resulting population dynamics (Moilanen and Hanski 2001). The matrix population modeling framework that we applied to marine metapopulations addresses this gap in the quantitative treatment of connectivity. In particular, the damping ratio provides a well-defined link between pair-wise connection strengths specified in the connectivity matrix and population-level processes. More generally, we propose that the mathematical tools provided by the matrix-modeling framework (e.g., analyses of sensitivity and elasticity; Caswell 2001:206257) are useful and powerful tools for understanding metapopulation connectivity.

\section{ACKNOWLEDGMENTS}

Funding for this work was provided by an ISAT Linkage grant from the Royal Society of New Zealand to J. Shima and E. Noonburg, and a Marsden grant from the Royal Society of New Zealand to J. Shima. Concepts and ideas presented in this paper also benefited from useful discussions with S. Swearer, and from constructive comments provided by three anonymous reviewers. This paper is a contribution from the Victoria University Coastal Ecology Lab (VUCEL).

\section{Literature Cited}

Aiken, C. M., S. A. Navarrette, M. I. Castillo, and J. C. Castilla. 2007. Along-shore larval dispersal kernels in a numerical ocean model of the central Chilean coast. Marine Ecology Progress Series 339:13-24.

Airoldi, L. 2003. The effects of sedimentation on rocky coast assemblages. Oceanography and Marine Biology Annual Review 41:161-236.

Bode, M., K. Burrage, and H. P. Possingham. 2008. Using complex network metrics to predict the persistence of metapopulations with asymmetric connectivity patterns. Ecological Modelling 214:201-209.

Calabrese, J. M., and W. F. Fagan. 2004. A comparisonshopper's guide to connectivity metrics. Frontiers in Ecology and the Environment 2:529-536.

Caley, M. J., M. H. Carr, M. A. Hixon, T. P. Hughes, G. P. Jones, and B. A. Menge. 1996. Recruitment and the local dynamics of open marine populations. Annual Review of Ecology and Systematics 27:477-500.
Caswell, H. 2001. Matrix population models: construction, analysis and interpretation. Sinauer Associates, Sunderland, Massachusetts, USA.

Cowen, R. K., C. B. Paris, and A. Srinivasan. 2006. Scaling of connectivity in marine populations. Science $311: 522-527$.

Cronin, J. T. 2007. From population sources to sieves: the matrix alters host-parasitoid source-sink structure. Ecology 88:2966-2976.

Cushing, D. H. 1975. Marine ecology and fisheries. Cambridge University Press, Cambridge, UK.

Denman, K. L., and T. M. Powell. 1984. Effects of physical processes on planktonic ecosystems in the coastal ocean. Oceanography and Marine Biology Annual Review 22:125168.

Doak, D. F., P. Marino, and P. M. Kareiva. 1992. Spatial scale mediates the influence of habitat fragmentation on dispersal success: implications for conservation. Theoretical Population Biology 41:315-336.

Elkin, C. M., and M. L. Reid. 2005. Low energy reserves and energy allocation decisions affect reproduction by Mountain Pine Beetles, Dendroctonus ponderosae. Functional Ecology 19:102-109.

Gaines, S. D., B. Gaylord, and J. L. Largier. 2003. Avoiding current oversights in marine reserve design. Ecological Applications 13(Supplement):S32-S46.

Gaylord, B., and S. D. Gaines. 2000. Temperature or transport? Range limits in marine species mediated solely by flow. American Naturalist 155:769-789.

Goodsell, P. J., and S. D. Connell. 2008. Complexity in the relationship between matrix composition and inter-patch distance in fragmented habitats. Marine Biology 154:117125.

Hanski, I. 2002. Metapopulation ecology. Oxford University Press, Oxford, UK.

Hanski, I., and O. E. Gaggiotti. 2004. Ecology, genetics and evolution of metapopulations. Elsevier Academic Press, San Diego, California, USA.

Haynes, K. J., and J. T. Cronin. 2004. Confounding of patch quality and matrix effects in herbivore movement studies. Landscape Ecology 19:119-124.

Hjort, J. 1914. The fluctuations in the great fisheries of northern Europe viewed in the light of biological research. Rapports Proces-Verbaux des Reunions - Conseil Permanent International pour l'Exploration de la Mer 20:1-228.

Kaplan, D. M. 2006. Alongshore advection and marine reserves: consequences for modeling and management. Marine Ecology Progress Series 309:11-24.

Kinlan, B. P., and S. D. Gaines. 2003. Propagule dispersal in marine and terrestrial environments: a community perspective. Ecology 84:2007-2020.

Kritzer, J. P., and P. F. Sale. 2004. Metapopulation ecology in the sea: from Levins' model to marine ecology and fisheries science. Fish and Fisheries 5:131-140.

Largier, J. L. 2003. Considerations in estimating larval dispersal distances from oceanographic data. Ecological Applications 13(Supplement):S71-S89.

Lasker, R. 1981. Marine fish larvae: morphology, ecology, and relation to fisheries. University of Washington Press, Seattle, Washington, USA.

Lebreton, J.-D. 1996. Demographic models for subdivided populations: the renewal equation approach. Theoretical Population Biology 49:291-313.

Lebreton, J.-D., and G. Gonzalez-Davila. 1993. An introduction to models of subdivided populations. Journal of Biological Systems 1:389-423.

MacArthur, R. H., and E. O. Wilson. 1967. The theory of island biogeography. Princeton University Press, Princeton, New Jersey, USA.

Mann, K. H., and J. R. N. Lazier. 1996. Dynamics of marine ecosystems. Blackwell Science, Malden, Massachusetts, USA. 
Matter, S. F. 2006. Changes in landscape structure decrease mortality during migration. Oecologia 150:8-16.

Meekan, M. G., J. H. Carleton, A. D. McKinnon, K. Flynn, and M. Furnas. 2003. What determines the growth of tropical reef fish larvae in the plankton: food or temperature? Marine Ecology Progress Series 256:193-204.

Minor, E. S., and D. L. Urban. 2008. A graph-theory framework for evaluating landscape connectivity and conservation planning. Conservation Biology 22:297-307.

Mitarai, S., D. A. Siegel, and K. B. Winters. 2008. A numerical study of stochastic larval settlement in the California Current system. Journal of Marine Sciences 69:295-309.

Moilanen, A., and I. Hanski. 2001. On the use of connectivity measures in spatial ecology. Oikos 95:147-151.

Morgan, L. E., and S. A. Shepherd. 2006. Population and spatial structure of two common temperate reef herbivores: abalone and sea urchins. Pages 205-234 in J. P. Kritzer and P. F. Sale, editors. Marine metapopulations. Academic Press, San Diego, California, USA.

Murphy, H. T., and J. Lovett-Doust. 2004. Context and connectivity in plant metapopulations and landscape mosaics: does the matrix matter? Oikos 105:3-14.

Nee, S. 2007. Metapopulations and their spatial dynamics. Pages 35-45 in R. May and A. McLean, editors. Theoretical ecology: principles and applications. Oxford University Press, New York, New York, USA.

Neubert, M. G., M. Kot, and M. A. Lewis. 1995. Dispersal and pattern formation in a discrete-time predator-prey model. Theoretical Population Biology 48:7-43.

O'Brien, D., M. Manseau, A. Fall, and M. J. Fortin. 2006. Testing the importance of spatial configuration of winter habitat for woodland caribou: an application of graph theory. Biological Conservation 130:70-83.

Okubo, A., and S. A. Levin. 2001. Diffusion and ecological problems. Springer, New York, New York, USA.

Paris, C. B., L. M. Cherubin, and R. K. Cowen. 2007. Surfing, spinning or diving from reef to reef: effects on population connectivity. Marine Ecology Progress Series 347:285-300.

Phillips, N. E., and J. S. Shima. 2006. Differential effects of suspended sediments on larval survival and settlement of New Zealand urchins (Evechinus chloroticus) and abalone (Haliotis iris). Marine Ecology Progress Series 314:149-158.

Pineda, J., J. A. Hare, and S. Sponaugle. 2007. Larval transport and dispersal in the coastal ocean and consequences for population connectivity. Oceanography 20:22-39.

Possingham, H. P., and J. Roughgarden. 1990. Spatial population dynamics of a marine organism with a complex life cycle. Ecology 71:973-985.

Revilla, E., T. Wiegand, F. Palomares, P. Ferreas, and M. Delibes. 2004. Effects of matrix heterogeneity on animal dispersal: from individual behavior to metapopulation-level parameters. American Naturalist 164:131-153.

Ricketts, T. H. 2001. The matrix matters: effective isolation in fragmented landscapes. American Naturalist 158:87-99.

Roff, D. 1977. Dispersal in dipterans - its costs and consequences. Journal of Animal Ecology 46:443-456.

Roughgarden, J., Y. Iwasa, and C. Baxter. 1985. Demographic theory for an open marine population with space-limited recruitment. Ecology 66:54-67.
Saunders, D. A., R. J. Hobbs, and C. R. Margules. 1991. Biological consequences of ecosystem fragmentation: a review. Conservation Biology 51:18-32.

Saura, S., and L. Pascual-Hortal. 2007. A new habitat availability index to integrate connectivity in landscape conservation planning: comparison with existing indices and application to a case study. Landscape and Urban Planning 83:91-103.

Shima, J. S., and S. E. Swearer. 2009. Larval quality is shaped by matrix effects: implications for connectivity in a marine metapopulation. Ecology 90:1255-1267.

Siegel, D. A., S. Mitarai, C. J. Costello, S. D. Gaines, B. E. Kendall, R. R. Warner, and K. B. Winters. 2008. The stochastic nature of larval connectivity among nearshore marine populations. Proceedings of the National Academy of Sciences USA 105:8974-8979.

Steele, J. H. 1989. The ocean "landscape." Landscape Ecology $3: 185-192$.

Teske, P. R., I. Papadopoulos, G. I. Zardi, C. D. McQuaid, M. T. Edkins, C. L. Griffiths, and N. P. Barker. 2007. Implications of life history for genetic structure and migration rates of southern African coastal invertebrates: planktonic, abbreviated and direct development. Marine Biology 152:697-711.

Thrush, S. F., J. E. Hewitt, V. J. Cummings, J. I. Ellis, C. Hatton, A. Lohrer, and A. Norkko. 2004. Muddy waters: elevating sediment input to coastal and estuarine habitats. Frontiers in Ecology and the Environment 2:299-306.

Tischendorf, L., and L. Fahrig. 2000. On the use and measurement of landscape connectivity. Oikos 90:7-19.

Tischendorf, L., and L. Fahrig. 2001. On the use of connectivity measures in spatial ecology. A reply. Oikos 95:152-155.

Treml, E. A., P. N. Halpin, D. Urban, and L. F. Pratson. 2008. Modeling population connectivity by ocean currents, a graph-theoretic approach for marine conservation. Landscape Ecology 23:19-36.

Urban, D., and T. Keitt. 2001. Landscape connectivity: a graph-theoretic perspective. Ecology 82:1205-1218.

Urban, D. L., E. S. Minor, E. A. Treml, and R. S. Schick. 2009. Graph models of habitat mosaics. Ecology Letters 12:260 273.

Vandermeer, J., and R. Caravajal. 2001. Metapopulation dynamics and the quality of the matrix. American Naturalist 158:211-220.

Vandermeer, J., and B. B. Lin. 2008. The importance of matrix quality in fragmented landscapes: understanding ecosystem collapse through a combination of deterministic and stochastic forces. Ecological Complexity 5:222-227.

Vos, C. C., P. W. Goedhart, and D. R. Lammertsma. and A. M. Spitzen-Van der Sluijs. 2007. Matrix permeability of agricultural landscapes: an analysis of movements of the common frog (Rana temporaria). Herpetological Journal 17: $174-182$.

Westerberg, L., Ö. Östman, and U. Wennergren. 2005. Movement effects on equilibrium distributions of habitat generalists in heterogeneous landscapes. Ecological Modelling 188:432-447.

Westerberg, L., and U. Wennergren. 2003. Predicting the spatial distribution of a population in a heterogeneous landscape. Ecological Modelling 166:53-65. 